\title{
Formation, electronic structure, and optical properties of self-assembled quantum-dot single-photon emitters in $\mathrm{Ga}(\mathrm{N}, \mathrm{As}, \mathrm{P})$ nanowires
}

\author{
M. Jansson $\odot,{ }^{1, *}$ L. Francaviglia $\odot,{ }^{2}$ R. La $\odot,{ }^{3}$ C. W. Tu $\odot,{ }^{3}$ W. M. Chen, ${ }^{1}$ and I. A. Buyanova ${ }^{1}$ \\ ${ }^{1}$ Department of Physics, Chemistry and Biology, Linköping University, SE-58183 Linköping, Sweden \\ ${ }^{2}$ Laboratoire des Matériaux Semiconducteurs, Institut des Matériaux, Ecole Polytechnique Fédérale de Lausanne, \\ 1015 Lausanne, Switzerland \\ ${ }^{3}$ Department of Electrical and Computer Engineering, University of California, San Diego, La Jolla, California 92093, USA
}

(Received 3 December 2019; revised manuscript received 26 February 2020; accepted 21 April 2020; published 15 May 2020)

\begin{abstract}
Quantum dots (QDs) formed in semiconductor nanowires (NWs) form a basis for studying interesting quantum phenomena and provide an exciting platform for various device applications, where in-depth understanding of the formation and electronic properties of such QDs is a requirement. In this work we present detailed investigations of structural, electronic, and optical properties of nitrogen-induced self-assembled QDs in novel $\mathrm{Ga}(\mathrm{N}, \mathrm{As}, \mathrm{P}) \mathrm{NWs}$. We show that the three-dimensional quantum confinement of the excitons caused by shortrange fluctuations in the $\mathrm{N}$ content leads to single-photon emission. We demonstrate that valence band character varies between the QD emitters and attribute it to a varying degree of hole localization within the QDs induced by fluctuations in the As/P ratio. These fluctuations in the chemical compositions are also believed to be at least partly responsible for the observed large variation in exciton lifetimes of the QDs, ranging between 0.5 and $10 \mathrm{~ns}$. The rather long lifetimes demonstrate the resilience of the QDs to nonradiative recombination-a merit for efficient single-photon emitters. Furthermore, we show that the principal axes of the formed QDs are oriented radially, normal to the NW side facets, in contrast to the N-induced QDs in GaNAs NWs which are primarily aligned along the NW axis. Our results, therefore, underline the impacts of the host alloy on the QD formation, which could provide an additional degree of freedom for band structure engineering in nanowires.
\end{abstract}

DOI: 10.1103/PhysRevMaterials.4.056005

\section{INTRODUCTION}

The III-V semiconductor nanowires (NWs) show a great promise for electronic and optoelectronic applications due to their high electron mobility and highly tunable and mostly direct band gap, in combination with the possibility of integration with $\mathrm{Si}$ and achieving waveguiding in NWs [1-7]. Additional advantages are provided by the possibility to embed quantum dots (QDs) into NWs, as it allows attaining the regime of full quantum confinement and thereby exploiting quantum phenomena. Two approaches are currently pursued for bottom-up fabrication of such hybrid QD-NW structures. The first one is based on deliberate QD formation in axial heterostructures formed by using different III-V materials [8-12], or via crystal phase engineering $[13,14]$. This approach allows precise control of QD properties by varying alloy compositions and quantum confinement, and was successfully implemented using various III-V alloys, such as AlGaN [8], AlGaAs [9], InAsP [10,11], and GaAsP [12]. Lateral sizes

\footnotetext{
*Corresponding author: mattias.jansson@liu.se
}

Published by the American Physical Society under the terms of the Creative Commons Attribution 4.0 International license. Further distribution of this work must maintain attribution to the author(s) and the published article's title, journal citation, and DOI. Funded by Bibsam. of the so-formed QDs and preferential orientation of their quantization axes are usually fixed by the NW diameter and its growth direction, respectively. The second approach relies on spontaneous self-assembly. Examples of such QDs include QDs formed on NW surfaces by Stranski-Krastanov growth [15-17], at the core-shell interfaces [18-21], or due to alloy fluctuations [22,23]. Self-assembled QDs are often smaller in size and have multiple (sometimes random) orientations of their principal (or high-symmetry) axes.

We have previously shown that the formation of selfassembled QDs is very efficient in novel GaNAs NWs, adding to numerous attractive properties of dilute nitride alloys [24-28]. The formation of these QDs is caused by clustering of $\mathrm{N}$ atoms, which leads to strong electron confinement facilitated by the giant bowing in the band-gap energy in dilute nitrides [22,29]. In contrast to self-assembled QDs in other material systems, spontaneously formed QDs in GaNAs NWs were found to be oriented along one preferred direction coinciding with the NW axis [29]. Their electronic structure was concluded to be governed by the local strain within the $\mathrm{N}$ clusters based on polarization-resolved magneto-optical measurements.

Introducing phosphorus into the alloy, i.e., forming the GaNAsP alloy, adds an additional degree of freedom for band-gap tailoring and lattice matching, making this material suitable for, e.g., intermediate band solar cell applications [30,31]. In this work, we show that alloying with $\mathrm{N}$ also causes formation of self-assembled QDs in GaNAsP NWs and 
investigate their structural, electronic, and optical properties. We found that, in stark contrast with the N-induced QDs in ternary GaNAs, the QDs formed in the GaNAsP NWs have their high-symmetry axis (the axis normal to the highsymmetry plane) aligned perpendicular to both the NW axis and the side facets of the NW. Moreover, though the emission energy of the QD emitters is determined by fluctuations in the N composition, as in the case of GaNAs NWs [29], their electronic structure is now controlled by local fluctuations in the As/P ratio that changes quantum confinement of the localized holes. The revealed QDs are also shown to act as single-photon emitters.

\section{EXPERIMENTAL DETAILS}

The studied GaNAsP NWs were fabricated by selfcatalyzed plasma-assisted molecular beam epitaxy (MBE). The growth was performed on (111) $\mathrm{Si}$ substrates using thermally cracked $\mathrm{PH}_{3}$ and $\mathrm{AsH}_{3}$ in combination with a solid $\mathrm{Ga}$ source and a $\mathrm{N}$ plasma. The $\mathrm{P}$ and $\mathrm{N}$ content in the group-V sublattice were estimated as $24 \%$ and $0.08 \%$, respectively [32]. The former was determined by energy dispersive $\mathrm{x}$-ray spectroscopy (EDXS), whereas the latter was deduced by analyzing photoluminescence (PL) data using the bandanticrossing model (BAC) [32]. The NWs have a length of approximately $2 \mu \mathrm{m}$ with a diameter of $100 \mathrm{~nm}$ [33]. Full details about the NW growth can be found in Ref. [34].

For characterization of individual NWs, they were mechanically transferred onto either another Si substrate or a carbon-copper transmission electron microscopy (TEM) grid. Cathodoluminescence (CL) spectroscopy was performed at an accelerating voltage of $5 \mathrm{keV}$ using the Rosa microscope from Attolight. Micro-PL ( $\mu$-PL) measurements were conducted in a backscattering geometry in a LHe-cooled coldfinger cryostat with a possibility to apply a magnetic field parallel to the light excitation/collection axis. Continuous wave (cw) excitation was performed using a 532-nm solidstate laser, and the PL emission was dispersed by a singlegrating monochromator and detected by a charge-coupled device (CCD). A pulsed Ti:sapphire laser with a repetition rate of $76 \mathrm{MHz}$ and a pulse width of $150 \mathrm{fs}$ was used as an excitation source in transient $\mu$-PL experiments, where the PL signal was detected by a streak camera coupled with a single-grating monochromator. In both cases the PL excitation and collection was performed using a $50 \times(0.5 \mathrm{NA})$ objective. For linear polarization-resolved PL measurements, a linearpolarization analyzer, which consisted of a rotatable half-wave plate and a fixed linear polarizer, was placed in front of the monochromator. The degree of linear PL polarization was calculated by $P=\left(I_{\max }-I_{\min }\right) /\left(I_{\max }+I_{\min }\right) \times 100$, where $I_{\max }\left(I_{\min }\right)$ is the maximum (minimum) PL intensity measured during a full $360^{\circ}$ rotation of the linear polarizer in the plane orthogonal to the direction of excitation/detection. The PL polarization was determined taking into account the dielectric mismatch between the NW and the ambient, which enhances the intensity of emitted light linearly polarized parallel to the NW growth axis [35]. Time-correlated single-photon counting (TCSPC) measurements were carried out in a Hanbury Brown and Twiss setup [36] with $\mathrm{cw}$ excitation provided by the 532-nm solid-state laser. The PL signal was detected by using two avalanche photodiodes in combination with two singlegrating spectrometers for signal filtering. All measurements were performed at temperatures in the range of 4-8 K.

\section{RESULTS AND DISCUSSION}

\section{A. Nitrogen-induced quantum-dot formation}

Figure 1(a) shows a $\mu$-PL spectrum acquired from a representative GaNAsP NW (the red curve). For easy reference, we also show the PL spectrum from a reference GaAsP NW (the black curve) with the same P/As incorporation ratio. As expected, alloying with nitrogen leads to a redshift of the emission due to the giant bowing of the conduction band in combination with exciton localization in the low-energy localized tail states [37]. The tail-state localization also leads to a spectral broadening [37]. Furthermore, numerous sharp PL lines appear on top of the broad PL background in the GaNAsP spectrum. The narrow linewidth (200-500 $\mu \mathrm{eV})$ of these transitions hints to three-dimensional (3D) exciton confinement, likely due to the formation of $\mathrm{N}$-induced QD-like states caused by short-range alloy fluctuations in GaNAsP. A similar effect has previously been observed in ternary GaNAs NWs [22,29].

To ascertain that the sharp PL lines stem from quantum emitters, TCSPC was performed on representative emission lines. Fig. 1(b) shows an autocorrelation histogram of the normalized coincidence counts for the exciton line shown in the inset. A clear antibunching dip is observed, with a measured value of the second-order coherence function at $t=0\left[g^{2}(0)\right]$ of 0.45 . The detected PL emission ( $\left.I_{\text {Total }}\right)$ is, however, a combination of the $\mathrm{QD}$ emission $\left(I_{\mathrm{QD}}\right)$ and the uncorrelated background emission, with a ratio of $\rho \equiv$ $I_{\mathrm{QD}} / I_{\text {Total }}=0.76$. For a perfect single-photon emitter, $g^{2}(0)=$ 0 , while the measured value $g_{\mathrm{m}}^{2}(0)$ increases with decreasing $\rho$ following the relation $g_{\mathrm{m}}^{2}(0)=1-\rho^{2}$. With $\rho=0.76$ a theoretical minimum value of $g_{\mathrm{m}}^{2}(0)=0.42$ [the gray dotted line in Fig. 1(b)] is found, which is very close to the measured value of 0.45 , meaning that within experimental error and accounting for the uncorrelated background, the investigated QD behaves as a single-photon emitter.

The localized nature of these emitters was further confirmed from CL measurements performed on individual GaNAsP NWs. Figure 1(c) reports the SEM and CL images of a single NW. The CL maps are energy filtered around 1.61, 1.69 , and $1.72 \mathrm{eV}$ with a bandwidth of approximately $5 \mathrm{meV}$. Figure 1(d) reports selected CL spectra extracted from the CL maps in Fig. 1(c) at the locations indicated by the dashed lines. The data are displayed after subtracting the broad background signal originating from the weakly localized excitons. Figure 1(d) shows that the sharp emission lines also present in the PL spectrum in Fig. 1(a) can be detected throughout the NW, with variable spectral positions along the NW. Consistently, the CL images in Fig. 1(c) contain spatially localized bright spots, roughly $70 \pm 20 \mathrm{~nm}$ in full width at half maximum, whose locations along the NW differ for different emission energies. These results prove that the GaNAsP NWs contain QD-like emitters that we ascribe to a spontaneous formation process due to nitrogen incorporation. 

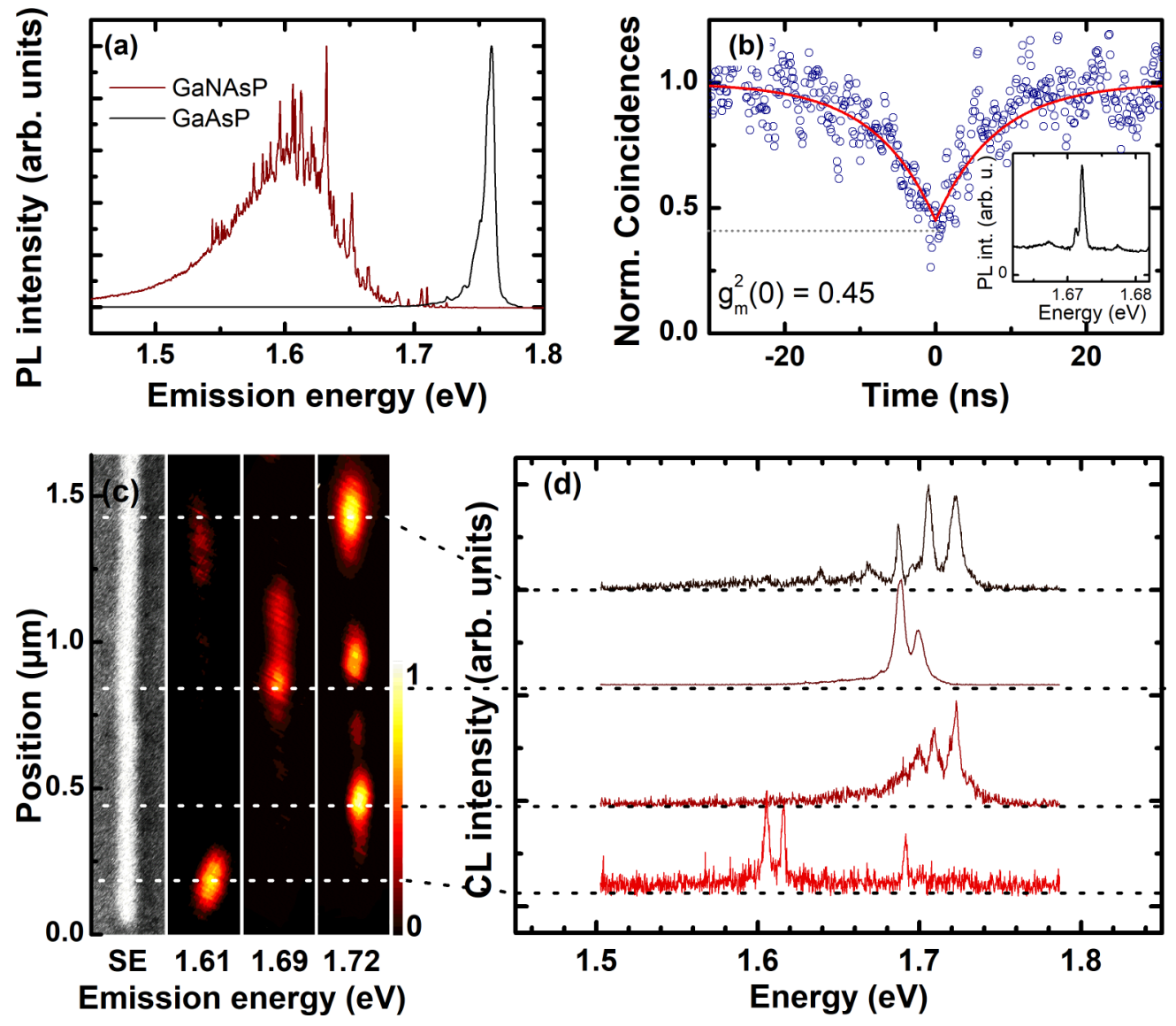

FIG. 1. (a) $\mu$-PL spectra measured from a representative single GaNAsP (the red curve) and GaAsP (the black curve) nanowire. (b) Normalized autocorrelation histogram (symbols) acquired from a single QD using TCSPC, together with a best fit using the function $f=1-C e^{-|t| / \tau}$ (red line). The gray dotted line represents an expected $\mathrm{g}^{2}(0)$ value, taking into account the ratio $(\rho=0.76)$ between the QD emission and the total emission. The inset shows the corresponding $\mu$-PL spectrum, containing the QD emission and an uncorrelated background emission. (c) Monochromatic CL images detected at the specified emission energies together with a secondary electron (SE) SEM micrograph of the NW (left). (d) CL spectra acquired from several locations of the same NW, as marked by the dashed lines. In (c,d), the broad background emission was subtracted from the CL spectra, to better visualize the QD emission.

\section{B. Electronic structure}

The electronic structure of the QDs is analyzed using polarization-resolved magneto- $\mu \mathrm{PL}$ measurements performed in the backscattering geometry, with an applied magnetic field aligned parallel to the light excitation/collection axis. The measurements were performed on individual NWs lying on a Si substrate. Similar to the case of the N-induced QDs in GaNAs NWs [29], two types of QDs can be distinguished.

The first type exhibits spectral characteristics suggesting that the hole participating in the monitored excitons is of pure heavy-hole $(\mathrm{HH})$ character. This implies a large $\mathrm{HH}-\mathrm{LH}$ (light-hole) splitting such that the LH states are not occupied at low temperatures used in our experiments. The electronic structure of such QDs, without the application of an external magnetic field $B$, is shown schematically in Fig. 2(a). The exciton states contain two bright states $|M\rangle=| \pm 1\rangle$ (the solid lines), which are degenerate for QDs of high symmetry $\left(C_{3 v}\right.$ or higher), but may experience a small fine-structure splitting $(\Delta 1)$ when the symmetry is lower than $C_{3 v}$. The $| \pm 2\rangle$ states (denoted by the dotted lines) are completely dark without an external magnetic field and anisotropic exchange interaction.
Consistently, such QDs are found to emit a single PL line or a pair of orthogonally polarized PL components with a very small splitting $(\Delta 1<100 \mu \mathrm{eV})$ at $B=0 \mathrm{~T}$. Typical spectra of such $\mathrm{HH}$ excitons detected in linear polarization parallel $\left(I_{\|}\right)$and orthogonal $\left(I_{\perp}\right)$ to the NW axis are shown by the solid lines in Fig. 2(b), whereas the transformation of the PL spectra under an applied magnetic field is illustrated in Figs. 2(c) and 2(d). At 0 T, only a single peak corresponding to the degenerate $| \pm 1\rangle$ doublet is observed. It is weakly polarized parallel to the NW axis, due to a tilt of the QD high-symmetry axis away from the light detection axis (to be discussed below). With an applied magnetic field, the line splits into two components with comparable intensities, as expected for the $| \pm 1\rangle$ states. A weak PL line, which becomes visible at $B>0$ on the low-energy side of the PL spectra, stems from the $| \pm 2\rangle$ states as the corresponding transitions become partly allowed under an applied $B$. The data can be modeled assuming that the monitored $\mathrm{HH}$ excitons stem from a QD with the high-symmetry axis tilted $60^{\circ}$ away from the direction of the applied magnetic field in the plane perpendicular to the NW axis; see below for a more detailed discussion. The results of the modeling, using the spin Hamiltonian described 


\section{Pure HH}

(a)

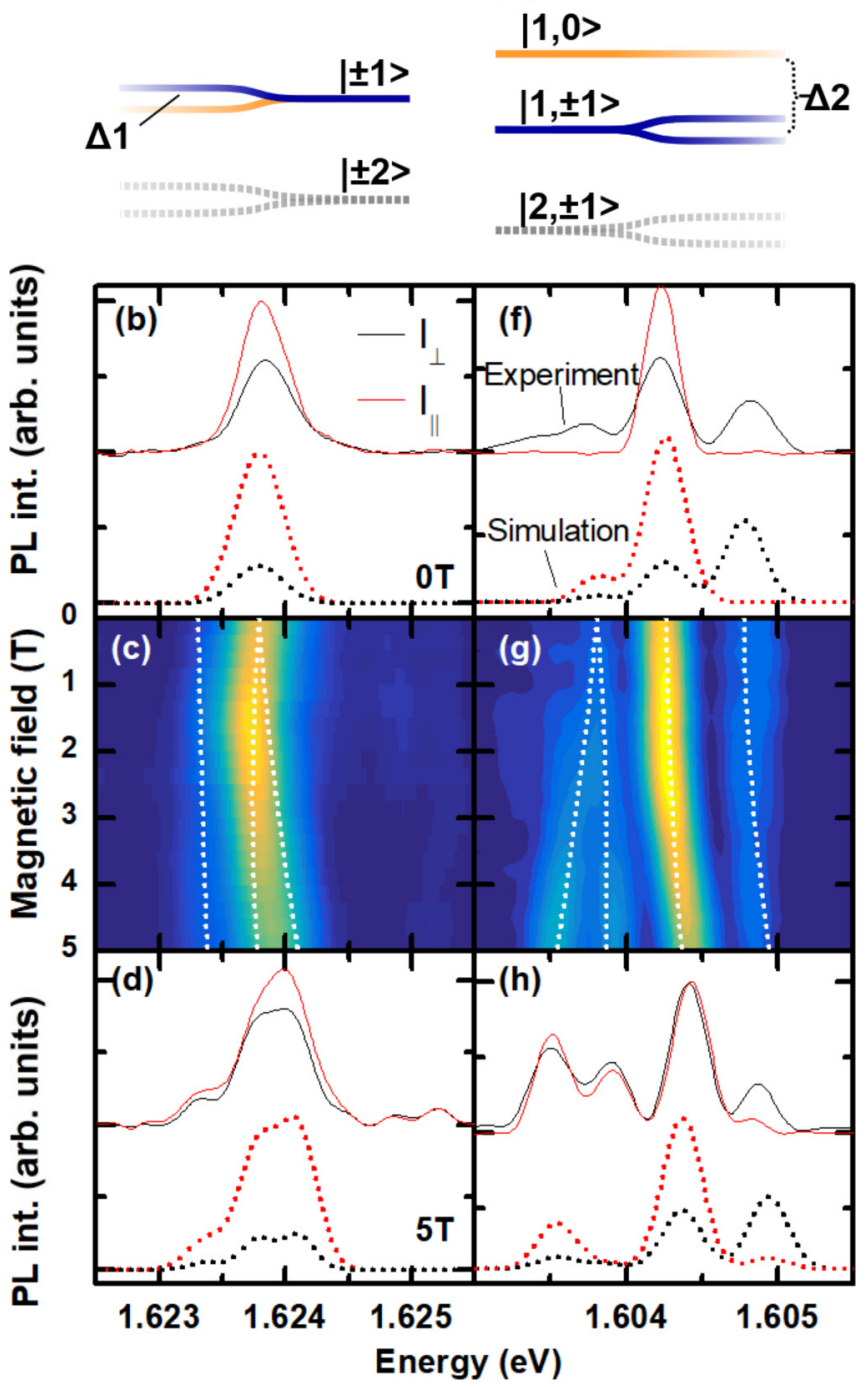

FIG. 2. Energy levels of excitons with a pure HH (a) and mixed LH-HH (e) character at $B=0$. Bright and dark states are shown by the solid and dotted lines, respectively. In (a) the LH states are not shown, for simplicity, due to a large LH-HH splitting such that they are not occupied at the measurement temperatures. In (e), only the dark states which are expected to mix with the bright states are included. The energy splitting $\Delta 1$ represents a splitting caused by lowering of the QD symmetry below $C_{3 v} . \Delta 2$ is the energy splitting between the $|1,0\rangle$ and $|1, \pm 1\rangle$ states, which is present for QDs with symmetry of $C_{3 v}$ or lower. Measured (solid lines) and simulated (dotted lines) $\mu$-PL spectra at $B=0 \mathrm{~T}$ of a representative pure HH (b) and mixed LH-HH (f) QD. The corresponding spectra at $B=5 \mathrm{~T}$ are shown in $(\mathrm{d}, \mathrm{h})$. The black and red curves are the $\mu$-PL spectra detected with linear polarization parallel $\left(I_{\|}\right)$and orthogonal $\left(I_{\perp}\right)$ to the NW axis. (c,g) PL images of the total $\mu$-PL intensity as a function of magnetic field and emission energy. The dotted lines represent the simulated energy of the exciton states.

in Ref. [29], are shown by the dotted lines in Figs. 2(b)-2(d), and are in good agreement with the experimental data. The linewidths of the simulated spectra are chosen to match those of the measured spectra. The simulations yield the in-plane (out-of-plane) exciton Landé $g$ value of $1.2(2.5)$ and the diamagnetic coefficient $(\gamma)$ of $4 \mu \mathrm{eV} / \mathrm{T}^{2}$. We note that some pure HH QD emitters do not exhibit linear polarization at $B=$ $0 \mathrm{~T}$. Their behavior under applied magnetic fields (not shown) can be simulated assuming that the high-symmetry axis of the corresponding QDs is parallel to the applied magnetic field.

The second type represents QDs of which the hole character is a mixture of LH and HH states. In this case, the bright exciton states consist of the singlet $|J, M\rangle=|1,0\rangle$ and the doublet $|1, \pm 1\rangle$ states, that are split by a rather large energy ( $\Delta 2$ ) typically exceeding $100 \mu \mathrm{eV}[29]$ —see Fig. 2(e). Moreover, five dark states $(J=2)$ exist at lower energies. In Fig. 2(e) only the $|2, \pm 1\rangle$ states, which mix with the bright states even without an external magnetic field, are shown. The $\mu$-PL spectra of such QDs contain a pair of orthogonally polarized lines corresponding to the bright $|1, \pm 1\rangle$ doublet and $|1,0\rangle$ singlet states-see Fig. 2(f). A small feature observed at the lower energies stems from the dark $|2, \pm 1\rangle$ states. With increasing $B$, the polarization of the $|1, \pm 1\rangle$ peak decreases, while the polarization of the $|1,0\rangle$ peak remains almost unchanged. Moreover, the exciton component originating from the dark states increases in intensity and splits into two peaks. These features are well reproduced by the simulation of a mixed LH-HH QD with its high-symmetry axis tilted by $60^{\circ}$ away from the axis of the applied magnetic field. The simulation results are shown by the dotted lines in Figs. 2(f)2(h). The best fit to the experimental data is obtained assuming $\gamma=3.5 \mu \mathrm{eV} / \mathrm{T}^{2}$, the electron $g$ value $\mathrm{g}_{e}=1.4$, and isotropic $(\mathrm{K})$ and anisotropic (L) Luttinger-Kohn parameters of -1.1 and 0.4 , respectively. We note a discrepancy between the simulated and measured peak intensity of one of one of the "dark" states in Fig. 2(h), where the simulated oscillator strength is underestimated. The reason for this discrepancy is not well understood, but we speculate that a further reduction of the QD symmetry, e.g., through shear strain, may further mix the bright and dark states, thereby affecting the oscillator strength.

Having established the coexistence of the two types of QDs with pure $\mathrm{HH}$ and mixed $\mathrm{LH}-\mathrm{HH}$ character, it is important to understand the cause for the difference in their electronic structure. Both types show comparable distributions within PL energies-see Figs. 3(a) and 3(b). This means that the local nitrogen concentration within the QDs, which is likely the primary cause of the large variation in the emission energy, is not the main factor determining the hole character. This is somewhat surprising considering that in ternary GaNAs NWs the LH-HH splitting and the hole character of the Ninduced QDs are largely determined by the local nitrogen concentration, due to an increased tensile strain within the QDs emitting at lower energies (and, therefore, with a higher local N concentration) [29]. The current GaNAsP NWs have, however, a significantly (by about one order of magnitude) lower $\mathrm{N}$ composition so that the local strain may not be the dominant factor. Moreover, we have previously shown that in the quaternary GaNAsP NWs, nitrogen incorporates mainly in As-rich regions [32]. This will cause compensation of the nitrogen-induced local strain, effectively reducing the straininduced spectral dependence of the hole character. To account for the variation of the LH-HH splitting in the investigated GaNAsP alloy we recall that, though fluctuations in the $\mathrm{N}$ 


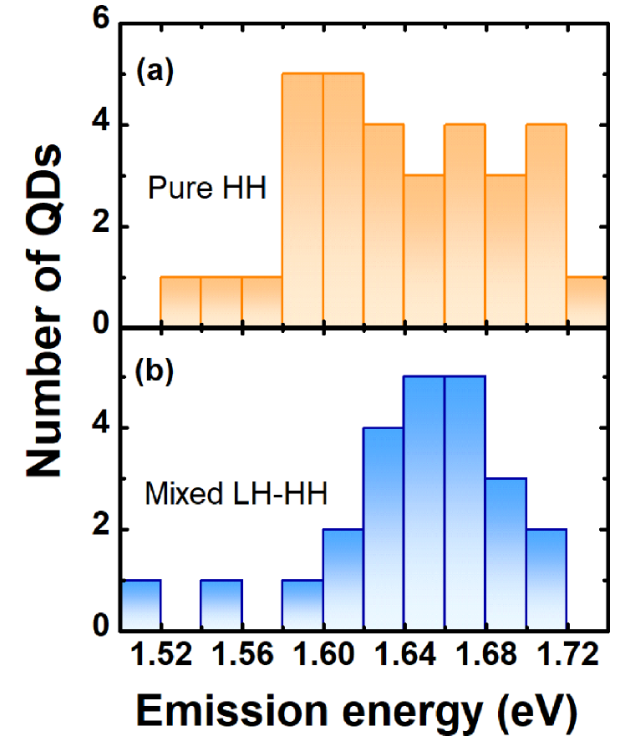

FIG. 3. istograms showing spectral distributions of QDs with pure $\mathrm{HH}$ (a) and mixed LH-HH (b) hole character, respectively.

content do not significantly affect the valence band (VB) edge of the alloy, fluctuations in the P/As ratio may lead to quantum confinement of holes in As-rich regions due to the type-I band alignment between $\mathrm{GaAs}$ and $\mathrm{GaP}$ [38].

The LH-HH splitting within these regions and the hole character of the excitons will then depend on the local P/As ratio and the extent of spatial localization. Following this reasoning, we suggest that, while in ternary GaNAs alloys the electronic structure of QDs is strongly dependent on the local nitrogen concentration, in quaternary GaNAsP alloys the electronic structure is dependent on the local concentrations of all group- $\mathrm{V}$ elements.

\section{Orientation of the QDs}

In some material systems, self-assembled QDs in NWs may be directly observed by, e.g., scanning transmission electron microscopy, allowing a straightforward investigation of their lateral position and orientation [18,23,39,40]. For the presently studied $\mathrm{N}$-induced QDs, however, such electron microscopy studies have not been successful due to the very low concentration of nitrogen. Instead, the orientation of QDs can be deduced by investigating polarization characteristics of the QD emission, by measuring the PL intensity as a function of the angular position of the linear polarization analyzer relative to the NW axis. This is possible because the polarization direction of the light emitted from recombination
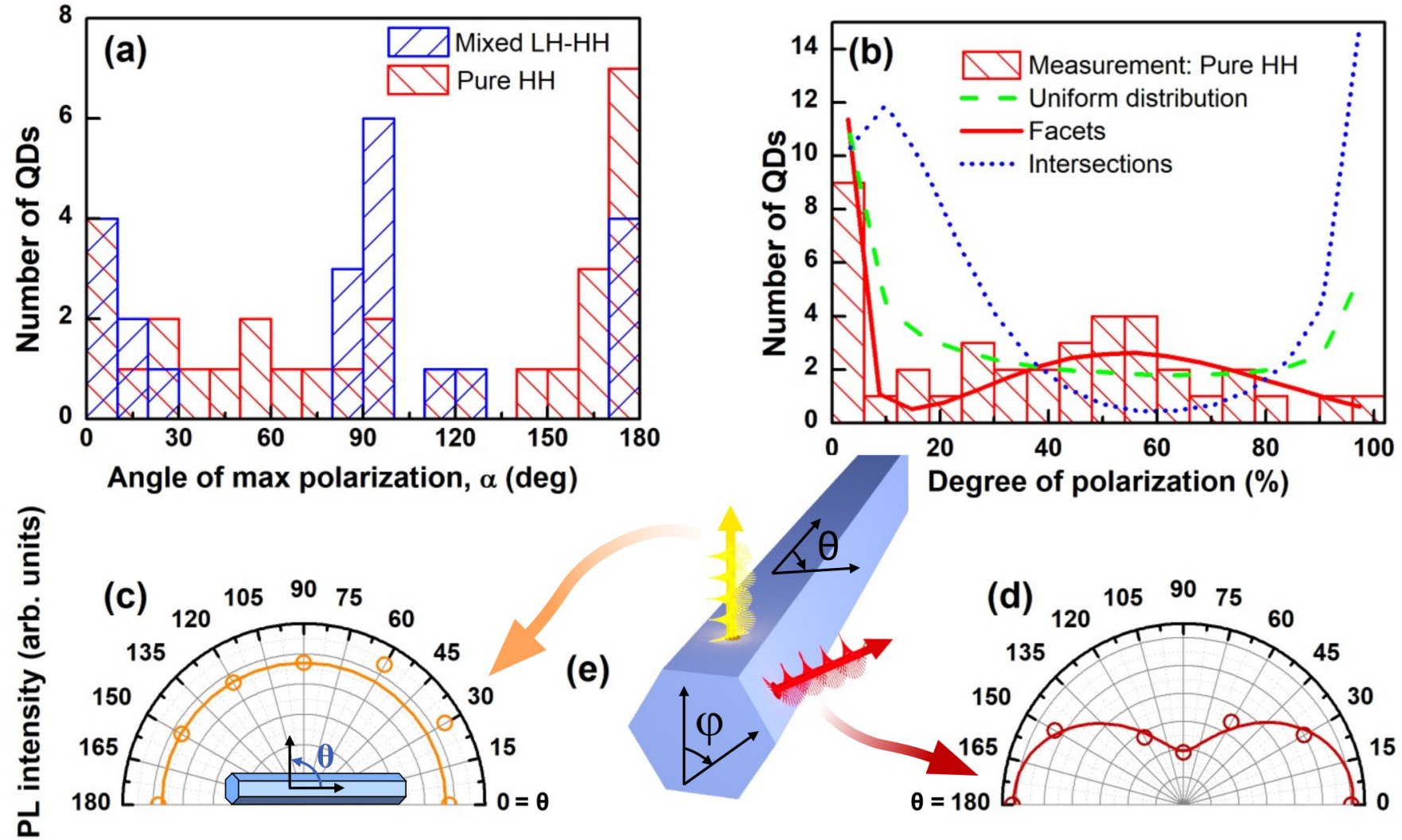

(e)

FIG. 4. (a) Statistical distributions of the angle of the maximum PL polarization, $\alpha$, for pure HH (red) and mixed LH-HH (blue) QDs, where $\alpha=0^{\circ}$ corresponds to light polarized parallel to the NW axis. (b) A histogram showing the degree of PL polarization for the investigated QDs, taking as an example pure HH QDs. Polar plots of the measured (symbols) and simulated (solid lines) PL intensity for a pure HH QD with the dominant quantization axis directed orthogonally to the NW facet with $\varphi=0^{\circ}$ (c) and $\varphi=60^{\circ}$ (d). The data were obtained by rotating a linear polarizer by the angle $\theta$ from the NW axis. (e) Schematic illustration of the definitions of the angles $\theta$ and $\varphi$ with respect to the NW, where the two arrows show the directions of the dominant quantization axes for the QDs with $\varphi=0^{\circ}$ and $\varphi=60^{\circ}$. 
of QD excitons is dictated by the QD high-symmetry axis. A statistical analysis of angle $\alpha$ at which the PL intensity reaches its maximum value is shown in Fig. 4(a), for the pure HH (red) and mixed LH-HH (blue) QDs, where they are distinguished on the basis of observation of two orthogonally polarized lines in the PL spectrum of the latter type. The two orthogonally polarized emission peaks from mixed LH-HH QDs (blue) are primarily polarized parallel and perpendicular to the NW axis, i.e., with $\alpha=0^{\circ}, 90^{\circ}$, and $180^{\circ}$. This distribution is consistent with two possible QD orientations: with the highsymmetry axis either (i) along or (ii) perpendicular to the NW growth axis. We note that for mixed LH-HH emitters with the high-symmetry axis along the experimental light collection (backscatter) axis, unpolarized light is expected from the $|1, \pm 1\rangle$ transitions while light from the $|1,0\rangle$ transition will not be detectable. Therefore, such emitters do not show the two orthogonally polarized lines and cannot be distinguished as mixed LH-HH QDs using the polarization-resolved data. They are thus excluded from the present analysis. For the pure HH QDs (red), while the preference of $\alpha=0^{\circ}$ and $180^{\circ}$ is seen, QDs polarized at all angles are observed. Moreover, some of the $\mathrm{HH}$ lines are not polarized in the measurement geometry (not included in the statistical analysis). This is inconsistent with the case (i), where we would rather expect light polarized perpendicular to the NW axis $\left(\alpha=90^{\circ}\right)$. On the other hand, the finding is in good agreement with the model (ii) that the QD principal axes are aligned radially. In this case, light emitted parallel to the detection path in the backscattering geometry would be completely circularly polarized, or with a small polarization in a random direction due, e.g., to sheer strain. Light emitted radially in other directions would be detected as polarized parallel to the NW, with varying polarization degree depending on the radial angle $\varphi$ [Fig. 4(e)], as indeed observed experimentally. Assuming that both types of the QDs have the same orientation, we therefore conclude that the QDs are aligned with the high-symmetry axis directed radially in the plane perpendicular to the NW axis.

To investigate whether the QDs are formed with some preference in the radial angle $\varphi$, we analyze the degree of polarization $P$ of the pure HH QDs. A histogram of the measured $P$ values is shown in Fig. 4(b) and exhibits two maxima corresponding to $P=0 \%$ and 55\%. Polar plots of the PL intensity for the corresponding QD emitters are shown by the open circles in Figs. 4(c) and 4(d), respectively. Knowing that for pure HH QDs of high symmetry the emitted light is circularly polarized with the electric-field vector lying in the high-symmetry plane of the QD, these polar plots can be simulated assuming that $\varphi=0^{\circ}$ and $60^{\circ}$, respectively, i.e., assuming that the high-symmetry QD axis is aligned orthogonally to the NW facets-see Fig. 4(e) for a schematic illustration. The same conclusion follows from the expected distribution of the polarization degree of the HH QD emitters simulated for possible radial orientations of their highsymmetry axis: (i) a uniform distribution over all angles $\varphi-$ shown by the green dashed line in Fig. 4(b); (ii) aligned through the intersections of the NW side facets-the blue dotted line in Fig. 4(b); and (iii) aligned orthogonally to the NW facets-the red solid line in Fig. 4(b). Clearly, a good agreement with the experimental data is found only in the last
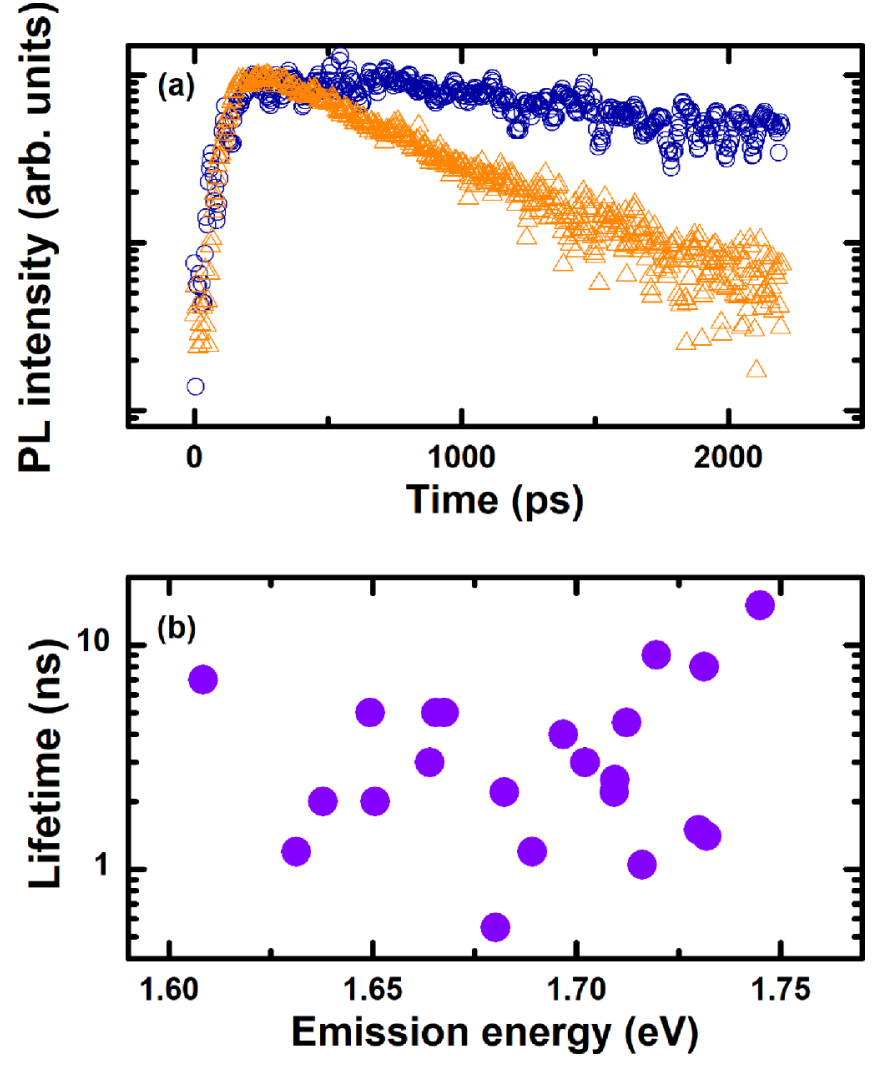

FIG. 5. (a) Representative PL decays of two QD emitters. (b) Exciton lifetimes of the QD emitters, as deduced from singleexponential fitting of the corresponding PL transients.

case, which suggests that the formed QD have the quantization axes oriented perpendicularly to the NW side facets. This conclusion is also supported by the magneto-PL data. It is interesting to note that this direction of the QD high-symmetry axis in the studied GaNAsP NWs essentially differs from the N-induced QDs in GaNAs NWs, where the high-symmetry axis is aligned primarily along the NW axis. Obviously, the host alloy has a strong effect on the QD properties. Though the exact physical mechanism responsible for this effect requires further studies, we speculate that the change in QD orientation upon $\mathrm{P}$ incorporation may be related to variations in the $\mathrm{P} / \mathrm{As}$ ratio, e.g., to a spontaneous radial gradient in the P/As ratio often observed in vapor-liquid-solid grown ternary III-V semiconductor NWs [41-44], such as GaAsP [41].

\section{Exciton lifetimes}

The lifetimes of various QDs were investigated using timeresolved $\mu$-PL. Figure 5(a) shows examples of PL decay curves measured from representative QDs, after subtraction of the broad background component. By fitting them with a single exponential function, the exciton lifetimes can be deduced and are given by the symbols in Fig. 5(b). It is evident that the lifetimes exhibit a substantial variation and are rather long, significantly longer than what is usually observed for self-assembled QDs in, e.g., the GaAs/AlAs [45,46] or the InAs/GaAs [47-49] material systems where the exciton lifetime is found to be below $1 \mathrm{~ns}$. Three effects can account 
for this behavior. Firstly, it could be caused by differences in spatial confinements of the electron and hole within the QD, reducing overlap of their wave functions. Indeed, since the giant bowing in the band-gap energy in dilute nitrides mainly occurs due to the downshift of the conduction band edge, fluctuations in the $\mathrm{N}$ composition will cause strong electron confinement. On the other hand, the hole within the exciton is either bound to the electron by Coulomb interaction or is confined due to fluctuations in the P/As ratio. The fact that the measured longest lifetime is close to the radiative lifetime of excitons in bulk GaNAs [50] supports this scenario. It can also account for the lack of the spectral dependence and a large scatter between the measured values, as the hole localization, which determines the overlap of $e / h$ wave functions and exciton lifetime, is not the decisive factor that governs the emission energy. Secondly, for mixed LH-HH QDs the oscillator strength of the QD emitters can also be reduced due to mixing between the bright $(J=1)$ and dark $(J=2)$ states. This effect is expected to be important if the QD symmetry is lower than $T_{d}$, and the degree of mixing will be strongly dependent on the strength and anisotropy of the exchange interaction, as well as on the local strain and crystal field the QD experiences. Thirdly, there could be a variation between QDs in the influence of nonradiative recombination processes via defects or surface states that shorten exciton lifetimes. At present, it remains unclear which of the aforementioned three mechanisms is dominating and is mainly responsible for the lifetime variation, which requires further in-depth studies.

\section{CONCLUSIONS}

In summary, we have shown that nitrogen incorporation in GaNAsP NWs causes the formation of QDs, due to the short-range fluctuations in the $\mathrm{N}$ composition that leads to large fluctuations in the conduction band edge. The localized origin of the formed QD emitters is independently confirmed from spatially resolved CL measurements. According to the time correlation measurements, the QDs act as single-photon emitters. This is evidence of single-photon emission from the $\mathrm{N}$-induced self-assembled QDs in dilute nitride NWs. The electronic structure of the formed QDs was investigated by employing polarization-resolved (magneto-) $\mu$-PL measurements. The hole in the QD excitons is found to have either pure $\mathrm{HH}$ or mixed $\mathrm{LH}-\mathrm{HH}$ character, which is attributed to a varying degree of hole localization due to fluctuations in the As/P incorporation ratio. Changes in the hole localization combined with a varying degree of mixing between the dark and bright exciton states, apart from nonradiative recombination, are suggested to cause a large variation in the exciton lifetimes deduced from $\mu$-PL transient measurements. Remarkably, we find that the high-symmetry axis of the QDs is directed in the radial direction, normal to the NW side facets, which is in stark contrast to the previously studied QDs in ternary GaNAs NWs. Our results, therefore, underline an important role of the host alloys in the QD formation, possibly providing an additional degree of freedom in engineering of self-assembled QDs embedded in NWs.

\section{ACKNOWLEDGMENTS}

The authors wish to thank A. Fontcuberta i Morral for collaboration during the cathodoluminescence measurements. Financial support by Linköping University through the Professor Contracts, the Swedish Research Council (Grant No. 2016-05091), the Swedish Energy Agency (Grant No. P40119-1), and the Swedish Government Strategic Research Area in Materials Science on Functional Materials at Linköping University (Faculty Grant SFO-Mat-LiU No. 2009 00971) is greatly appreciated. L.F. acknowledges funding from the Swiss National Foundation through the NCCR QSIT.
[1] G. Otnes and M. T. Borgström, Towards high efficiency nanowire solar cells, Nano Today 12, 31 (2017).

[2] S. W. Eaton, A. Fu, A. B. Wong, C.-Z. Ning, and P. Yang, Semiconductor nanowire lasers, Nat. Rev. Mater. 1, 16028 (2016).

[3] Y. Li, F. Qian, J. Xiang, and C. M. Lieber, Nanowire electronic and optoelectronic devices, Mater. Today 9, 18 (2006).

[4] H. J. Joyce, Q. Gao, H. H. Tan, C. Jagadish, Y. Kim, J. Zhou, L. M. Smith, H. E. Jackson, J. M. Yarrison-Rice, P. Parkinson, and M. B. Johnston, III-V semiconductor nanowires for optoelectronic device applications, Prog. Quantum Electronics. 35, 23 (2011).

[5] R. Yan, D. Gargas, and P. Yang, Nanowire photonics, Nat. Photonics 3, 569 (2009).

[6] P. C. McIntyre and A. Fontcuberta i Morral, Semiconductor nanowires: To grow or not to grow? Mater. Today Nano $\mathbf{9}$, 100058 (2019).

[7] L. Güniat, P. Caroff, and A. Fontcuberta i Morral, Vapor phase growth of semiconductor nanowires: Key developments and open questions, Chem. Rev. 119, 8958 (2019).
[8] J. Renard, R. Songmuang, C. Bougerol, B. Daudin, and B. Gayral, Exciton and biexciton luminescence from single GaN/AlN quantum dots in nanowires, Nano Lett. 8, 2092 (2008).

[9] J. Heinrich, A. Huggenberger, T. Heindel, S. Reizenstein, S. Höfling, L. Worschech, and A. Forchel, Single photon emission from positioned GaAs/AlGaAs photonic nanowires, Appl. Phys. Lett. 96, 211117 (2010).

[10] M. T. Björk, C. Thelander, A. E. Hansen, L. E. Jensen, M. W. Larsson, L. R. Wallenberg, and L. Samuelson, Fewelectron quantum dots in nanowires, Nano Lett. 4, 1621 (2004).

[11] E. D. Minot, E. Kelkensberg, M. van Kouwen, J. A. van Dam, L. P. Kouwenhoven, V. Zwiller, M. T. Borgström, O. Wunnicke, M. A. Verheijen, and E. P. A. M. Bakkers, Single quantum dot nanowire LEDs, Nano Lett. 7, 367 (2007).

[12] M. T. Borgström, V. Zwiller, E. Müller, and A. Imamoglu, Optically bright quantum dots in single nanowires, Nano Lett. 5, 1439 (2005). 
[13] K. A. Dick, C. Thelander, L. Samuelson, and P. Caroff, Crystal phase engineering in single InAs nanowires, Nano Lett. 10, 3494 (2010).

[14] N. Akopian, G. Patriarche, L. Liu, J.-C. Harmand, and V. Zwiller, Crystal phase quantum dots, Nano Lett. 10, 1198 (2010).

[15] E. Uccelli, J. Arbiol, J. R. Morante, and A. Fontcuberta i Morral, InAs quantum dot arrays decorating the facets of GaAs nanowires, ACS Nano 4, 5985 (2010).

[16] M. Heiss, B. Ketterer, E. Uccelli, J. R. Morante, J. Arbiol, and A. Fontcuberta i Morral, In(Ga)As quantum dot formation on group-III assisted catalyst-free InGaAs nanowires, Nanotechnology 22, 195601 (2011).

[17] X. Yan, X. Zhang, X. Ren, H. Huang, J. Guo, X. Guo, M. Liu, Q. Wang, S. Cai, and Y. Huang, Growth of InAs quantum dots on GaAs nanowires by metal organic chemical vapor deposition, Nano Lett. 11, 3941 (2011).

[18] M. Heiss, Y. Fontana, A. Gustafsson, G. Wüst, C. Magen, D. D. O'Regan, J. W. Luo, B. Ketterer, S. Conesa-Boj, A. V. Kuhlmann, J. Houel, E. Russo-Averchi, J. R. Morante, M. Cantoni, N. Marzari, J. Arbiol, A. Zunger, R. J. Warburton, and A. Fontcuberta i Morral, Self-assembled quantum dots in a nanowire system for quantum photonics, Nat. Mater. 12, 439 (2013).

[19] P. Corfdir, Y. Fontana, B. van Hattem, E. Russo-Averchi, M. Heiss, A. Fontcuberta i Morral, and R. T. Phillips, Tuning the g-factor of neutral and charged excitons confined to selfassembled (Al,Ga)As shell quantum dots, Appl. Phys. Lett. 105, 223111 (2014).

[20] L. Mancini, Y. Fontana, S. Conesa-Boj, I. Blum, F. Vurpillot, L. Francaviglia, E. Russo-Averchi, M. Heiss, J. Arbiol, A. Fontcuberta i Morral, and L. Rigutti., Three-dimensional nanoscale study of $\mathrm{Al}$ segregation and quantum dot formation in GaAs/AlGaAs core-shell nanowires, Appl. Phys. Lett. 105, 243106 (2015).

[21] Y. Fontana, P. Corfdir, B. van Hattem, E. Russo-Averchi, M. Heiss, S. Sonderegger, C. Magen, J. Arbiol, R. T. Phillips, and A. Fontcuberta i Morral, Exciton footprint of self-assembled AlGaAs quantum dots in core-shell nanowires, Phys. Rev. B. 90, 075307 (2014).

[22] S. Filippov, M. Jansson, J. E. Stehr, J. Palisaitis, P. O. A. Persson, F. Ishikawa, W. M. Chena, and I. A. Buyanova, Strongly polarized quantum-dot-like light emitters embedded in GaAs/GaNAs core/shell nanowires, Nanoscale 8, 15939 (2016).

[23] T. Shi, H. E. Jackson, L. M. Smith, N. Jiang, Q. Gao, H. H. Tan, C. Jagadish, C. Zheng, and J. Etheridge, Emergence of localized states in narrow GaAs/AlGaAs nanowire quantum well tubes, Nano Lett. 15, 1876 (2015).

[24] Y. Puttisong, I. A. Buyanova, A. J. Ptak, C. W. Tu, L. Geelhaar, H. Riechert, and W. M. Chen, Room-temperature electron spin amplifier based on $\mathrm{Ga}(\mathrm{In}) \mathrm{NAs}$ alloys, Adv. Mater. 25, 738 (2013).

[25] Y. Puttisong, X. J. Wang, I. A. Buyanova, L. Geelhaar, H. Riechert, A. J. Ptak, C. W. Tu, and W. M. Chen, Efficient room-temperature nuclear spin hyperpolarization of a defect atom in a semiconductor, Nat. Commun. 4, 1751 (2013).

[26] X. J. Wang, I. A. Buyanova, F. Zhao, D. Lagarde, A. Balocchi, X. Marie, C. W. Tu, J. C. Harmand, and W. M. Chen, Roomtemperature defect-engineered spin filter based on a nonmagnetic semiconductor, Nat. Mater. 8, 198 (2009).
[27] S. Chen, Y. Huang, D. Visser, S. Anand, I. A. Buyanova, and W. M. Chen, Room-temperature polarized spin-photon interface based on a semiconductor nanodisk-in-nanopillar structure driven by few defects, Nat. Commun. 9, 3575 (2018).

[28] S. Chen, M. Jansson, J. E. Stehr, Y. Huang, F. Ishikawa, W. M. Chen, and I. A. Buyanova, Dilute nitride nanowire lasers based on a GaAs/GaNAs core/shell structure, Nano Lett. 17 1775 (2017).

[29] M. Jansson, F. Ishikawa, W. M. Chen, and I. A. Buyanova, $\mathrm{N}$-induced quantum dots in $\mathrm{GaAs} / \mathrm{Ga}(\mathrm{N}, \mathrm{As})$ core/shell nanowires: symmetry, strain, and electronic structure, Phys. Rev. Appl. 10, 044040 (2018).

[30] H. Jussila, P. Kivisaari, J. Lemettinen, T. Tanaka, and M. Sopanen, Two-Photon Absorption in $\mathrm{GaAs}_{1-x-y} \mathrm{P}_{y} \mathrm{~N}_{x}$ Intermediate-Band Solar Cells, Phys. Rev. Appl. 3, 054007 (2015).

[31] Y. J. Kuang, K. M. Yu, R. Kudrawiec, A. V. Luce, M. Ting, W. Walukiewicz, and C. W. Tu, GaNAsP: An intermediate band semiconductor grown by gas-source molecular beam epitaxy, Appl. Phys. Lett. 102, 112105 (2013).

[32] M. Jansson, S. Chen, R. La, J. E. Stehr, C. W. Tu, W. M. Chen, and I. A. Buyanova, Effects of nitrogen incorporation on structural and optical properties of GaNAsP nanowires, J. Phys. Chem. C 121, 7047 (2017).

[33] M. Jansson, L. Francaviglia, R. La, R. Balagula, J. E Stehr, C. W Tu, A. Fontcuberta I Morral, W. M Chen, and I. A Buyanova, Increasing $\mathrm{N}$ content in GaNAsP nanowires suppresses the impact of polytypism on luminescence, Nanotechnology $\mathbf{3 0}$, 405703 (2019).

[34] R. La, J. L. Pan, F. Bastiman, and C. W. Tu, Selfcatalyzed $\mathrm{Ga}(\mathrm{N}) \mathrm{AsP}$ nanowires and GaAsP/GaNAsP core-shell nanowires grown on $\mathrm{Si}$ (111) by gas-source molecular beam epitaxy, J. Vac. Sci. Technol., B: Microelectron. Nanometer Struct.-Process., Meas., Phenom. 34, 02 L108 (2016).

[35] H. E. Ruda and A. Shik, Polarization-sensitive optical phenomena in semiconducting and metallic nanowires, Phys. Rev. B 72 , 115308 (2005).

[36] R. Hanbury Brown and R. Q. Twiss, Correlation between photons in two coherent beams of light, Nature 177, 27 (1956).

[37] For a review of dilute nitrides, see, e.g., Physics and Applications of Dilute Nitrides, edited by I. A. Buyanova and W. M. Chen (Taylor \& Francis, New York, 2004).

[38] M. E. Pistol and C. E. Pryor, Band structure of coreshell semiconductor nanowires, Phys. Rev. B 78, 115319 (2008).

[39] H. A. Fonseka, A. V. Velichko, Y. Zhang, J. A. Gott, G. D. Davis, R. Beanland, H. Liu, D. J. Mowbray, and A. M. Sanchez, Self-formed quantum wires and dots in GaAsP-GaAsP coreshell nanowires, Nano Lett. 19, 4158 (2019).

[40] S. Y. Woo, M. Bugnet, H. P. T. Nguyen, Z. Mi, and G. A. Botton, Atomic ordering in InGaN alloys within nanowire heterostructures, Nano Lett. 15, 6413 (2015).

[41] C. Chen, S. Shehata, C. Fradin, R. LaPierre, C. Couteau, and G. Weihs, Self-directed growth of AlGaAs core-shell nanowires for visible light applications, Nano Lett. 7, 2584 (2007).

[42] P. K. Mohseni, A. D. Rodrigues, J. C. Galzerani, Y. A. Pusep, and R. R. LaPierre, Structural and optical analysis of GaAsP/GaP core-shell nanowires, J. Appl. Phys. 106, 124306 (2009). 
[43] A. Fakhr, Y. M. Haddara, and R. R. LaPierre, Dependence of InGaP nanowire morphology and structure on molecular beam epitaxy growth conditions, Nanotechnology 21, 165601 (2010).

[44] Y. Guo, H.-Y. Xu, G. J. Auchterlonie, T. Burgess, H. J. Joyce, Q. Gao, H. H. Tan, C. Jagadish, H.-B. Shu, X.-S. Chen, W. Lu, Y. Kim, and J. Zou, Phase separation induced by Au catalysts in ternary InGaAs nanowires, Nano Lett. 13, 643 (2013).

[45] C. Heyn, C. Strelow, and W. Hansen, Excitonic lifetimes in single GaAs quantum dots fabricated by local droplet etching, New J. Phys. 14, 053004 (2012).

[46] P. A. Dalgarno, J. M. Smith, J. McFarlane, B. D. Gerardot, K. Karrai, A. Badolato, P. M. Petroff, and R. J. Warburton, Coulomb interactions in single charged self-assembled quantum dots: Radiative lifetime and recombination energy, Phys. Rev. B 77, 245311 (2008).
[47] G. Wang, S. Fafar, D. Leonard, J. E. Bowers, J. L. Merz, and P. M. Petroff, Time-resolved optical characterization of InGaAs/GaAs quantum dots, Appl. Phys. Lett. 64, 2815 (1994).

[48] F. Adler, M. Geiger, A. Bauknecht, F. Scholz, H. Schweizer, M. H. Pilkuhn, B. Ohnesorge, and A. Forchel, Optical transitions and carrier relaxation in self assembled InAs/GaAs quantum dots, J. Appl. Phys. 80, 4019 (1996)

[49] J. Siegert, S. Marcinkevičius, and Q. X. Zhao, Carrier dynamics in modulation-doped InAs/GaAs quantum dots, Phys. Rev. B 72, 085316 (2005).

[50] I. A. Buyanova, W. M. Chen, G. Pozina, J. P. Bergman, B. Monemar, H. P. Xin, and C. W. Tu, Mechanism for low-temperature photoluminescence in GaNAs/GaAs structures grown by molecular-beam epitaxy, Appl. Phys. Lett. 75, 501 (1999). 